\title{
Alignment of recombination sites in Hin-mediated site-specific DNA recombination
}

\author{
Ivan P.G. Moskowitz, ${ }^{1,2}$ Karen A. Heichman, ${ }^{1}$ and Reid C. Johnson ${ }^{1,3,4}$ \\ ${ }^{1}$ Department of Biological Chemistry, University of California at Los Angeles School of Medicine, Los Angeles, California \\ 90024-1737 USA; ${ }^{3}$ Molecular Biology Institute, University of California, Los Angeles, California 90024 USA
}

The Hin site-specific recombination system normally promotes inversion of DNA between two recombination sites in inverted orientation. We show that the rate of deletion of DNA between two directly repeated recombination sites is 10-300 times slower than inversion between sites in their native configuration as measured in vivo and in vitro, respectively. In vitro studies have shown that the deletion reaction has the same requirement for Fis, a recombinational enhancer, and DNA supercoiling as the inversion reaction. These requirements, together with the finding that the deletion products are interlinked once suggest that the deletion synaptic complex is similar to the invertasome intermediate that generates inversion. The inefficiency of the deletion reaction is not a function of a reduced ability to recognize or synapse recombination sites in direct orientation. Not only do these substrates support an efficient knotting reaction, but directly repeated recombination sites with symmetric core sequences also invert efficiently. These findings demonstrate that the recombination sites are preferentially assembled into the invertasome structure with the sites aligned in the configuration for inversion regardless of their starting orientation. We propose that the dynamics of a supercoiled DNA molecule biases the geometric assembly of specific intermediates. In the case of Hin-mediated recombination, inversion is overwhelmingly preferred over deletion because DNA supercoiling favors a specific alignment of DNA strands in the synaptic complex.

[Key Words: Hin; Fis; recombinational enhancer; site-specific recombination; DNA strand exchange; DNA supercoiling]

Received March 15, 1991; revised version accepted June 18, 1991.

The product of Hin-mediated site-specific recombination is the inversion of a 995-bp DNA segment in the Salmonella chromosome. Inversion of DNA between the two recombination sites switches the orientation of the promoter that transcribes the $H 2$ flagellin gene and the repressor of the unlinked $H 1$ flagellin gene, resulting in the alternate expression of the $H 1$ and $H 2$ flagellins (Silverman and Simon 1980; Zieg and Simon 1980). Scott and Simon (1982) demonstrated that Hin-mediated recombination in vivo resulted in deletion of the intervening DNA when the orientation of one of the recombination sites is reversed on a plasmid substrate. However, deletion of DNA located between the directly repeated recombination sites occurred much less efficiently than inversion between recombination sites in their native orientation. The related Gin and Cin recombinases invert a segment of DNA containing tail fiber genes in phage $\mathrm{Mu}$ and $\mathrm{Pl}$, respectively (Kamp et al. 1978; Iida et al. 1982). In each of these systems, the inversion reaction

${ }^{2}$ Present address: Department of Biochemistry, University of Wisconsin, Madison, Wisconsin 53706 USA.

${ }^{4}$ Corresponding author. is much more efficient than the deletion reaction (Kennedy et al. 1983; Plasterk et al. 1983). This bias in the relative efficiency of the two reactions is one of the hallmarks of the DNA invertase family.

The Hin-mediated DNA inversion reaction occurs efficiently in vitro with purified components (Johnson et al. 1986). Inversion requires a supercoiled DNA substrate containing the two recombination sites (hix sequences) and a recombinational enhancer sequence (Johnson and Simon 1985). The recombinational enhancer is a $63-\mathrm{bp}$ DNA segment that is located $99 \mathrm{bp}$ from the left recombination site in its native context but can function many kilobases away from either recombination site to promote inversion. Each recombination site consists of a 26-bp sequence in which two imperfect 12-bp half-sites are separated by a 2 -bp core where strand exchange occurs (Johnson and Simon 1985; Johnson and Bruist 1989). The relative orientation of the recombination sites with respect to each other is defined by their configuration in the Salmonella chromosome and the products of recombination. The hix sites in their native configuration (inverted) give rise to inversion of the intervening DNA, whereas in vivo analysis has shown that when one site is 
oriented in the reverse configuration (directly repeated) the intervening DNA segment is deleted (Scott and Simon 1982). Because of the symmetrical nature of the hix sequence, the molecular features that determine directionality of the site have been enigmatic.

Three proteins, Hin, Fis, and $\mathrm{HU}$, are required for efficient inversion in a wild-type plasmid substrate (Johnson et al. 1986). The Hin recombinase binds to each recombination site and catalyzes a staggered cleavage leaving the 2-bp core nucleotides as $3^{\prime}$-single-stranded ends. Hin remains covalently associated with the $5^{\prime}$ end of the cleavage site until the DNA is resealed (Johnson and Bruist 1989). Fis is a 98-amino-acid polypeptide that was first identified by its role in promoting site-specific DNA inversion (Johnson et al. 1986; Koch and Kahmann 1986). Fis binds to two sites in the recombinational enhancer (Bruist et al. 1987) and becomes colocalized with Hin bound at each recombination site to form a threelooped invertasome structure (see Fig. 1A) that is an intermediate in inversion (Heichman and Johnson 1990). Assembly of the invertasome may proceed by first pairing of the two recombination sites by Hin. It has been proposed that the association of the enhancer into the invertasome structure may be required to initiate rotation of the Hin subunits that are covalently attached to the core nucleotides after DNA cleavage (Heichman et al. 1991). The nonspecific DNA-binding protein HU is believed to help stabilize the invertasome by facilitating the looping of DNA. HU strongly stimulates inversion rates only when the enhancer is located close to a recombination site, where extreme bending of the DNA is required for invertasome assembly (Johnson et al. 1986; M. Haykinson and R. Johnson, unpubl.).

Initial in vitro experiments with partially purified extracts failed to detect deletions even though inversions were readily obtained (Johnson et al. 1984). We have now reinvestigated the deletion reaction using purified proteins, different substrates, and more sensitive detection procedures. We find that site-specific deletions by the Hin system can be catalyzed in vitro, albeit at a low rate. We have used the in vitro system to determine whether the deletion reaction is mechanistically related to inversion or whether it occurs by a separate pathway, and to address the mechanism responsible for the overwhelming preference of Hin to promote inversion over deletion. We find that the deletion reaction is probably very similar to the inversion reaction and that the low rate of the deletion reaction is not a function of inefficient recognition or reactivity of recombination sites in directly repeated configuration. Rather, the directionality bias is the result of preferential assembly of recombination sites into a particular geometric configuration irrespective of starting recombination site orientation. Strand exchange is attempted and results in inversion if the sequences of the core nucleotides at synapsis are identical. However, if the core nucleotides are not identical at synapsis, as in the case of directly repeated sites, knotted products are generated. These recombinant knots can be explained by an additional DNA strand rotation that would restore the complementarity of the core nucleotides and allow
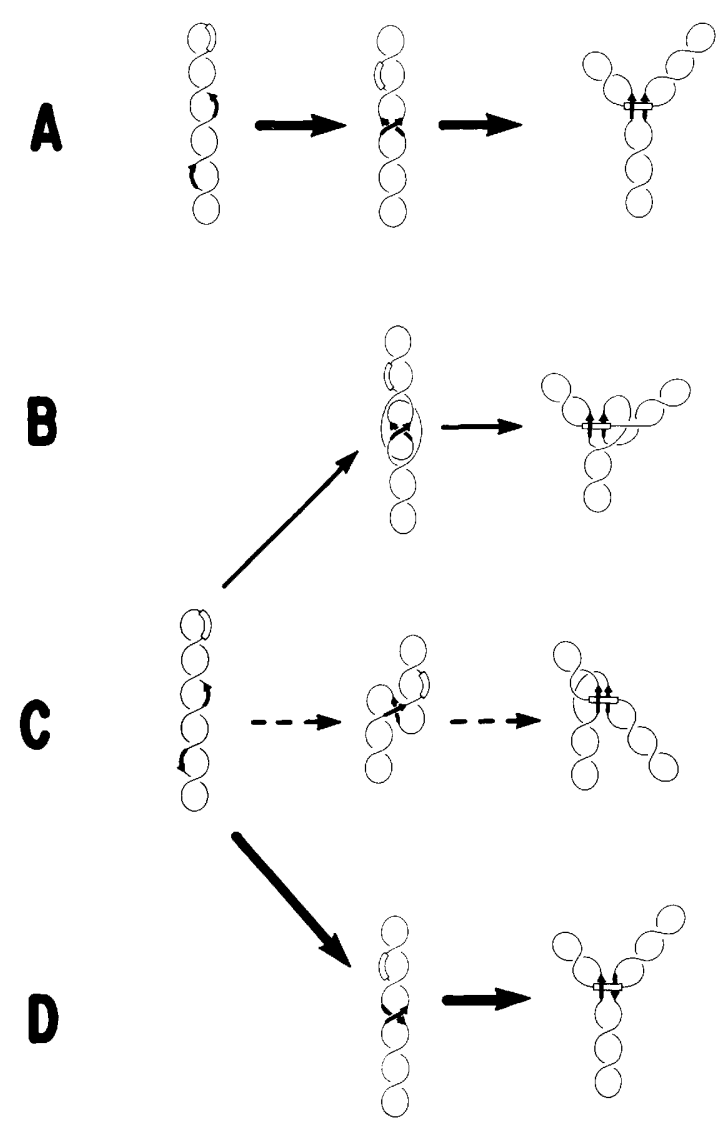

Figure 1. Pathways for assembly of recombination sites to generate inversion or deletion. In pathway $A$, the recombination sites are oriented in inverted (parallel) configuration with respect to each other as they are in the Salmonella chromosome. The recombination sites probably first associate on the supercoiled DNA molecule (paired hix structures) prior to assembly of the recombinational enhancer segment into the invertasome structure (Heichman and Johnson 1990). The paired hix structures are stabilized by interactions of Hin dimers bound to each of the recombination sites. The invertasome structure is stabilized by Hin-Fis interactions and DNA supercoiling, in addition to Hin-Hin interactions. Inversion of the segment of DNA containing the enhancer occurs by double-strand cleavage followed by a $180^{\circ}$ rotation of DNA strands and ligation in the invertasome complex (Heichman et al., this issue). Pathways $B-D$ represent three potential routes of assembly of recombination complexes starting with recombination sites in directly repeated orientation. Pathways $B$ and $C$ are two different ways on a supercoiled DNA molecule in which the recombination sites could associate with the enhancer into a configuration to generate deletion after strand exchange. If strand rotation occurs in the clockwise direction, the deletion products will be interlinked once in pathway $B$ and unlinked (free) in pathway $C$. In pathway $D$, the recombination sites assemble into a structure that is identical to the invertasome in pathway $A$, except the recombination sites are aligned in reverse (antiparallel) orientation. Two rotations of DNA strands must accompany strand exchange to ligate the products (see Fig. 8). This results in a knot being formed in the DNA but no change in the primary sequence. The results in this paper suggest that pathway $B$ is the most likely mechanism for the inefficient deletion reaction that occurs between directly repeated recombination sites, whereas pathway $D$ is the preferred assembly of directly repeated recombination sites. 
for ligation. This results in a change in the topology of the DNA but not in the primary sequence. The results suggest further that the preferential alignment of sites within the recombination complex is directed by the topological constraints imposed by a supercoiled DNA molecule and not a molecular feature of the sites themselves. Recent studies with the Gin inversion system reach similar conclusions with regard to recombination site synapsis (Kanaar et al. 1990).

\section{Results}

\section{Hin-mediated deletion in vivo}

The relative in vivo rates of inversion between recombination sites in inverted orientation and deletion between recombination sites in direct repeat orientation have been measured. The different plasmid substrates containing recombination sites in inverted or directly repeated orientation were transformed into a strain containing the hin gene under lacPO control (see Materials and methods). Hin expression was induced in culture, and aliquots were removed at various times for plasmid isolation and analysis. Except for the relative orientation of their recombination sites (see Fig. 2), pMS634 (inverted repeat, IR) and pRJ858 (direct repeat, DR) are similar in structure. Figure 3 compares the rates of inversion in these two plasmids. These plasmids contain an enhancer located 868 and $694 \mathrm{bp}$ from the recombination sites. Deletions occur at $\sim 8 \%$ the rate of inversions under these in vivo conditions. In vivo recombination rates in pMS551 (IR) and pMS638 (DR) or pRJ974 (DR), which have their enhancers located at the native position $99 \mathrm{bp}$ from hixL (Fig. 2), were indistinguishable from pMS634 (IR) and pRJ858 (DR), respectively (Table 1 ; data not shown).

\section{Hin-mediated deletion in vitro}

The preference for inversions over deletions is even greater in vitro than in vivo. Figure $4 \mathrm{~A}$ shows the result of an in vitro Hin reaction with pRJ858 (DR). In this experiment deletions are detected by digesting the products of the reaction with a restriction enzyme that cleaves once within the vector and radiolabeling the ends with ${ }^{32} \mathrm{P}$. The resulting linear parental product $(5377 \mathrm{bp}$ ) and deletion product $(3752 \mathrm{bp}$ ) are visualized after electrophoresis and quantitated. After $60 \mathrm{~min}, 6 \%$ of the molecules have undergone deletion. In a parallel experiment, the rates of inversion in vitro was measured in pMS634 (IR) (Fig. 4B). We estimate that the rate of deletion in vitro is $<0.3 \%$ of the rate of inversion using these substrates.

\section{Requirements for deletion formation}

To determine whether the deletion reaction requires the same reaction components as inversion, we have assayed for deletions under conditions that affect the efficiency

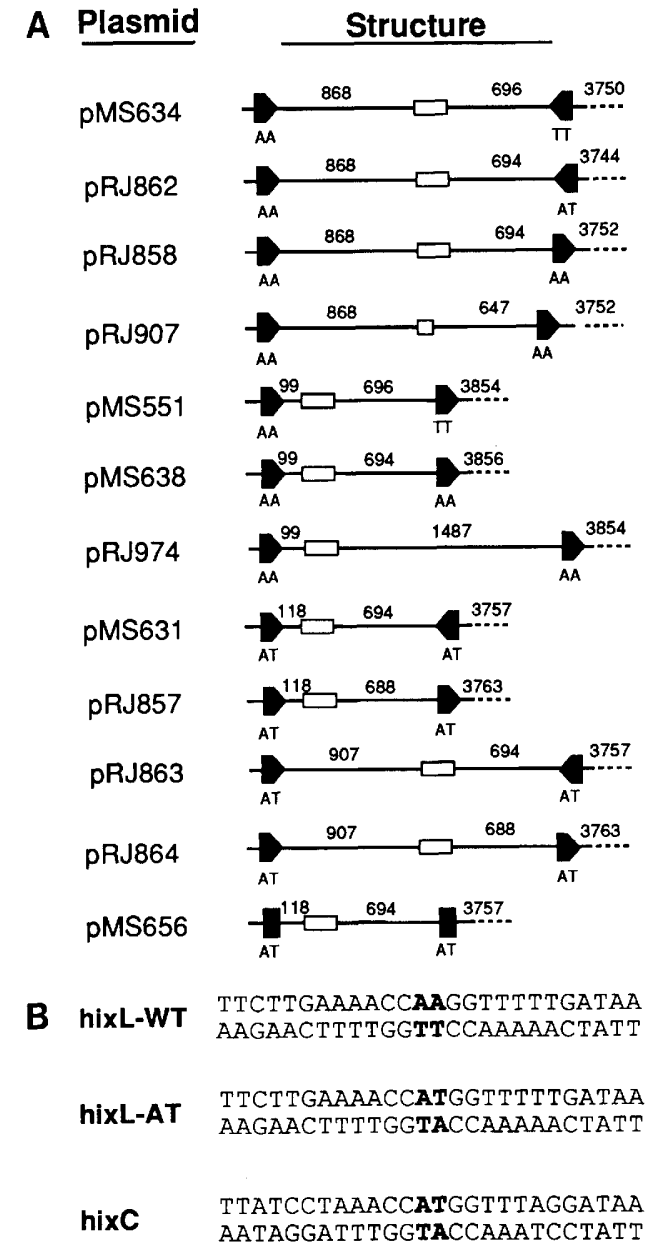

Figure 2. Structure of the plasmid substrates used in this study. $(A)$ The solid arrowheads denote the recombination sites, with AA or TT indicating the wild-type core sequence of hixL$W T$ and AT indicating the mutant core sequence present in hix $L-A T$. The relative orientations of the recombination sites are denoted by the direction of the arrowheads: Directly repeated recombination sites have arrowheads pointing in the same direction; inverted recombination sites have arrowheads oriented toward each other. Because of the symmetric nature of the hix $C$ recombination sites, their relative orientation is indistinguishable. The location of the 63-bp enhancer segment is indicated by the open rectangle. pRJ907 is missing $87 \mathrm{bp}$, which includes part of the enhancer segment. The number of base pairs between the center of the recombination sites and the boundaries of the enhancer are given along with the length of the vector sequences (above the broken lines) as measured between the center of the two recombination sites. $(B)$ Sequences of the wild-type hix $L$ recombination site (hix $L-W T$ ), a mutant hix $L$ recombination site (hix $L-A T)$, and a perfectly symmetric recombination site $($ hixC $)$ that is a consensus sequence of all the known DNA invertase systems related to Hin. The bold nucleotides denote the core residues where DNA strand exchange takes place.

of inversion. No deletion products were detected in reactions with pRJ858 (DR) in the absence of Fis and in substrates lacking a functional enhancer [pRJ907 (DR); Table 2]. A requirement of DNA supercoiling for dele- 


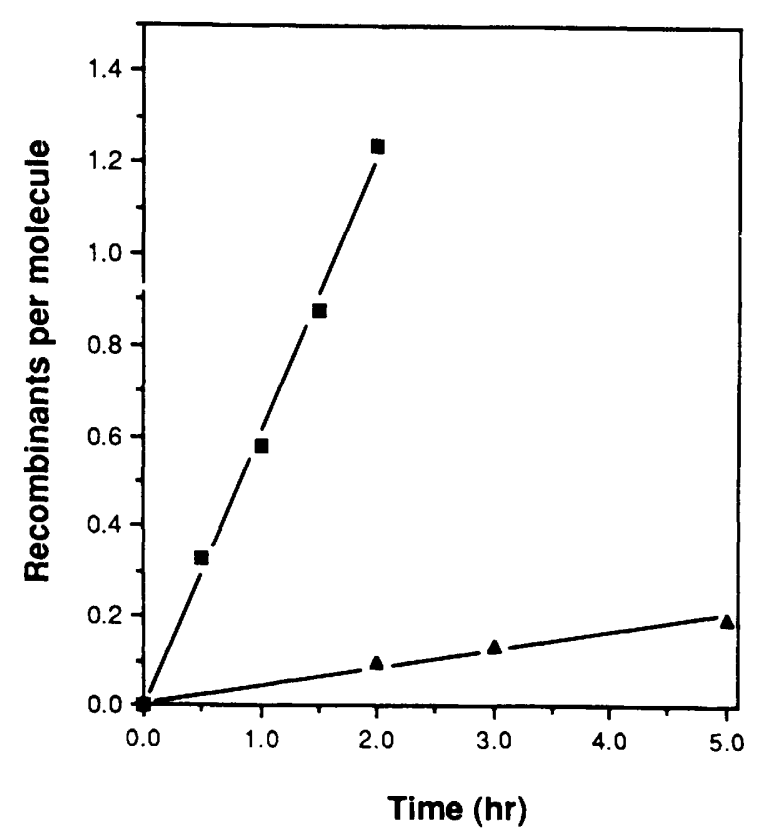

Figure 3. Rates of inversion vs. deletion in vivo. Expression of the hin gene in RJ2626 containing pMS634 (IR) or pMS858 (DR) was initiated by the addition of IPTG. At various times after Hin induction, aliquots were removed, plasmid DNA was prepared, and inversion or deletion was measured. The results are plotted as recombinants (inversions in pMS634 (IR), $\mathbf{\square}$; or deletions in pMS858 (DR), $\mathbf{\Delta}$ ] per substrate molecule as a function of time after hin induction.

tion was demonstrated with pRJ858 (DR) that had been relaxed previously with topoisomerase (Table 2 ). No recombinants were detected, demonstrating that superhelicity is necessary to generate deletions as has been observed for inversion (Johnson et al. 1986).

HU was also demonstrated to play a role in the Hinmediated deletion. pMS638 (DR) and pRJ974 (DR) contain the enhancer beginning $99 \mathrm{bp}$ from the center of one of the hix sites, which is its native position with respect to hixL (Fig. 2). These plasmids only supported deletions at $10-20 \%$ of the rate of pRJ858 (DR) with $100 \mathrm{ng}$ of $\mathrm{HU}$ per 0.1 pmole of DNA, the amount added in standard inversion reactions (Table 2; data not shown). There was almost no detectable reaction in the absence of $\mathrm{HU}$ and demonstrably greater reaction with increasing HU. The addition of three times the amount of HU that is optimal for inversion resulted in deletion rates in pMS638 (DR) approaching those measured for pRJ858 (DR). In contrast, deletion formation in pRJ858 (DR) displayed only about a twofold stimulation by $\mathrm{HU}$ and was slightly inhibited with increasing levels. Thus, HU stimulates deletion to a greater extent when the enhancer is located close to a recombination site than when it is located $>700 \mathrm{bp}$ from either recombination site. This resembles the pattern observed for inversion (Johnson et al. 1986), although deletion formation displays a stronger requirement for HU than inversion under conditions where the enhancer and a recombination site are positioned at the native position.

\section{Deletion products are singly catenated}

The requirement for the enhancer, Fis, and DNA supercoiling suggests that deletions may be generated by a mechanism similar to that used to generate inversion. Recombination intermediates that could generate deletions, assuming that the interaction between Hin and Fis at the enhancer is analogous to that proposed for inversion, are depicted in the pathways in Figure $1, \mathrm{~B}$ and $\mathrm{C}$. Compared with the inversion complex (Fig. 1A), additional loops in DNA are required to orient the recombination sites into a configuration to generate deletion upon strand exchange. Analysis of DNA models suggests that due to the energetics of a supercoiled molecule, structure B would be more favorable than structure C. If the direction of strand rotation was exclusively in the clockwise direction, the product of recombination in structure B would be catenated with the resulting molecules interlinked once, but the deletion products generated from the intermediate depicted in structure $C$ would not be catenated. Topological analysis of Hin-mediated recombinant knots has shown that strand rotation is exclusively in the clockwise direction in the invertasome assembled on a supercoiled molecule (Heichman et al. 1991). A counterclockwise rotation of DNA strands in structure $\mathrm{C}$ would generate singly catenated products.

To determine whether the deletion products are catenated, the products of a reaction with pRJ858 (DR) were electrophoresed in an agarose gel and the DNA migrating as full-length supercoiled molecules was excised (Fig. 5A). This population will contain the parental (including knotted forms; see below) and any catenated deletion products; any free 1625-bp deletion circles will have migrated farther in the gel as denoted in Figure 5. After digestion with a restriction enzyme that cuts once

Table 1. Quantitation of in vivo inversion and deletion reactions

\begin{tabular}{lclcc}
\hline $\begin{array}{l}\text { Plasmid } \\
\text { substrate }\end{array}$ & $\begin{array}{l}\text { hix } \\
\text { structure }^{\mathrm{a}}\end{array}$ & $\begin{array}{l}\text { hix- } \\
\text { enhancer } \\
\text { spacing }^{\mathrm{b}}\end{array}$ & $\begin{array}{l}\text { Percent } \\
\text { inverted }^{\mathrm{c}}\end{array}$ & $\begin{array}{l}\text { Percent } \\
\text { deleted }^{\mathrm{d}}\end{array}$ \\
\hline pMS551 & WT $:$ inverted & close & 36.6 & $<2$ \\
pMS638 & WT $:$ direct & close & $<2$ & 23.2 \\
pMS631 & AT $:$ inverted & close & 31.2 & 18.8 \\
pRJ857 & AT $:$ direct & close & 32.1 & 22.2 \\
pMS634 & WT $:$ inverted & extended & 34.6 & $<1$ \\
pRJ858 & WT $:$ direct & extended & $<1$ & 19.4 \\
pRJ863 & AT $:$ inverted & extended & 34.2 & 23.1 \\
pRJ864 & AT $:$ direct & extended & 29.7 & 21.0 \\
pMS656 & hixC & close & 36.9 & $<2$ \\
\hline
\end{tabular}

${ }^{a}(\mathrm{WT})$ hix L-WT sites; (AT) hixL-AT sites present on the plasmid. Their relative orientation (inverted or direct repeats) is also given.

$\mathrm{b} /$ Close $) \leqslant 118 \mathrm{bp}$; (extended $) \geqslant 688 \mathrm{bp}$ between the enhancer and closest recombination site.

'The percentage of inverted molecules was determined after induction of Hin for 60 min except for pMS656, which was determined after $5 \mathrm{hr}$.

${ }^{\mathrm{d}}$ The percentage of molecules that had undergone deletion was determined $5 \mathrm{hr}$ after the induction of Hin. 


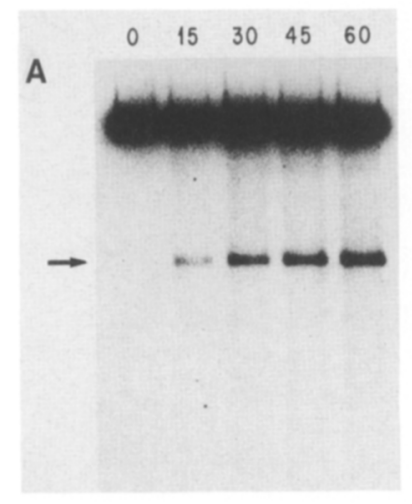

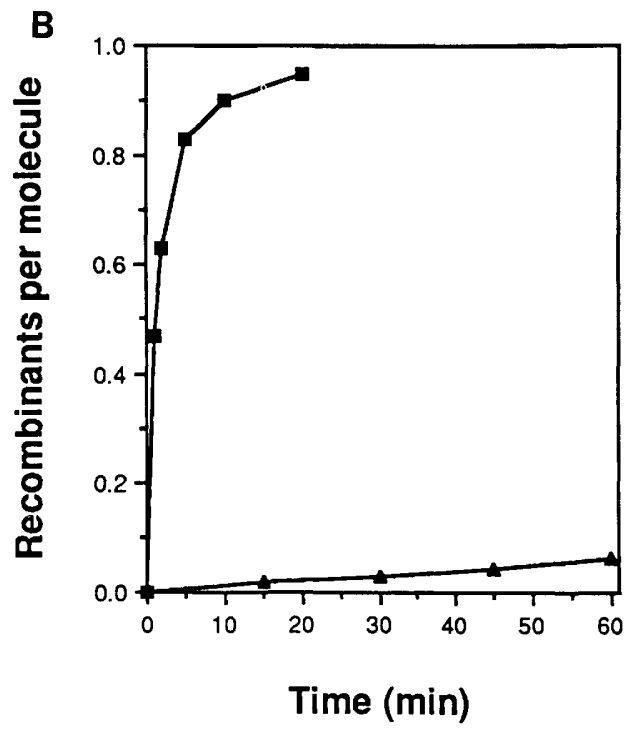

Figure 4. Rates of inversion vs. deletion in vitro. (A) pRJ858 (DR) was incubated with Hin, Fis, and HU. Aliquots were removed at the times indicated, digested with NdeI, which cuts once in the vector sequences, end-labeled with ${ }^{32} \mathrm{P}$, electrophoresed in an agarose gel, and autoradiographed. The arrow indicates the labeled deletion product. $(B)$ The amount of deletion products from $A$ was quantitated and plotted as deletion products $(\mathbf{\Delta})$ per substrate molecule as a function of time. For comparison, the number of inversions ( $\square$ ) per molecule in pMS634 (IR) as a function of time from a parallel experiment is shown. within the 1625-bp segment between the recombination sites, followed by end-labeling, the molecules were electrophoresed again and the products were detected by autoradiography (Fig. 5B). The number of deletion products obtained was nearly identical after gel isolation to the number obtained without prior gel isolation $(5.1 \%$ vs. $5.7 \%$, respectively), indicating that the products must be catenated.

Electron microscopy of deletion products that were coated with RecA was performed to determine the complexity of catenation (Krasnow et al. 1983). For this experiment we used pRJ864, which contains two hix L-AT

Table 2. Requirements for Hin-mediated site-specific deletion

\begin{tabular}{|c|c|c|}
\hline Reaction $^{a}$ & Substrate & $\begin{array}{l}\text { Relative number } \\
\text { of deletions }{ }^{b}\end{array}$ \\
\hline \multicolumn{3}{|l|}{ A } \\
\hline complete & pRJ858 & 1.0 \\
\hline no Fis & pRJ858 & $<0.02$ \\
\hline no enhancer & pRJ907 & $<0.02$ \\
\hline no supercoiling & pRJ858 & $<0.02$ \\
\hline \multicolumn{3}{|l|}{$\mathrm{B}$} \\
\hline complete & pRJ858 & 1.0 \\
\hline no HU & pRJ858 & 0.57 \\
\hline $3 \times \mathrm{HU}$ & pRJ858 & 0.73 \\
\hline complete & pMS638 & 0.17 \\
\hline no HU & pMS638 & 0.02 \\
\hline $3 \times \mathrm{HU}$ & pMS638 & 0.34 \\
\hline
\end{tabular}

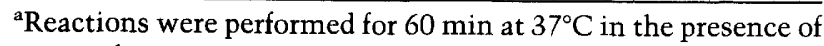
$70 \mathrm{ng}$ of Hin, $25 \mathrm{ng}$ of Fis, and $100 \mathrm{ng}$ of $\mathrm{HU}$ on 0.1 pmole of supercoiled plasmid substrate (complete) unless denoted otherwise. $(3 \times \mathrm{HU}) 300 \mathrm{ng}$ of $\mathrm{HU}$ was included in the reaction.

${ }^{b}$ The percent of plasmids containing deletions are presented relative to $\mathrm{pRJ} 858$ in complete reaction conditions. In experiment A, pRJ858 gave $5.2 \%$ deletions; in experiment $\mathrm{B}, \mathrm{pRJ} 858$ gave $8.6 \%$ deletions.

cpRJ858 was relaxed with topoisomerase I prior to reaction. sites in direct repeat orientation. This plasmid supports wild-type rates of deletion (see below) but does not knot extensively, which simplified the search for deletion products (Heichman et al., this issue; data not shown). To enrich for deletions, the products of a reaction with pRJ864 (DR) were nicked with DNase I and electropho-

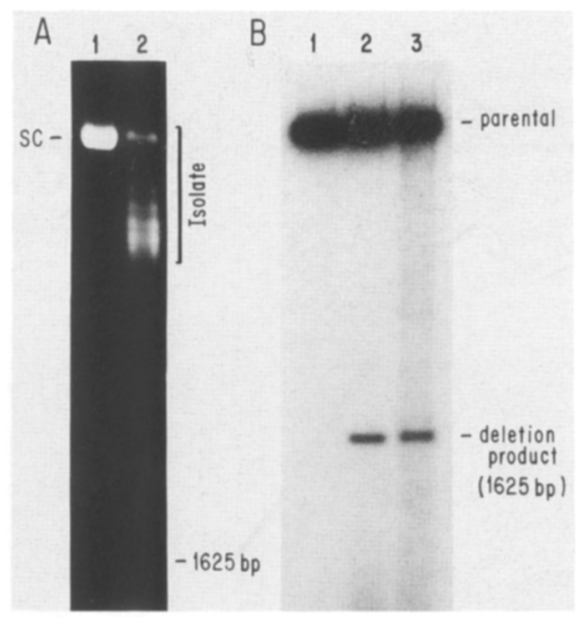

Figure 5. Hin-mediated deletion products are catenated. pRJ858 (DR) was reacted with Hin, Fis, and $\mathrm{HU}$ for $60 \mathrm{~min}$ and electrophoresed in an agarose gel $(A$, lane 2$)$ together with unreacted supercoiled DNA (lane 1). The location of a potential free 1625-bp supercoiled deletion product is indicated as determined by electrophoresing supercoiled plasmids of known size on the same gel. The DNA migrating in the region of the parental supercoiled (SC) and knotted forms, as indicated, was isolated, cleaved with HindIII, which cuts within the 1625-bp segment between the recombination sites, end-labeled with ${ }^{32} \mathrm{P}$, electrophoresed on a second agarose gel, and autoradiographed $(B$, lane 3). Lane $1(B)$ is HindIII-cut end-labeled pRJ858 (DR) that was not reacted with Hin; lane 2 is an aliquot of the same reaction used in $A$ that was digested with HindIII and end-labeled without gel isolation. The location of the 1625-bp labeled deletion product, as well as the parental DNA, is indicated. 
resed in an agarose gel. The DNA that migrated faster than parental relaxed molecules was purified, coated with RecA, and examined with the electron microscope. Two catenated deletion molecules were found, and each was interlinked once (Fig. 6). Ten deletion products generated with another plasmid that supports deletion [pMS631 (IR); see below] were also determined to be exclusively singly catenated.

\section{Substrates containing wild-type recombination sites in direct repeat orientation support efficient knotting}

In the work of Heichman et al. (this issue), it has been shown that the knotting of DNA by Hin is mechanistically related to recombination and is caused by multiple rotations of cleaved DNA strands prior to ligation. Although it only supports a low rate of deletion, pRJ858 (DR) is a very efficient substrate for Hin-mediated knotting. Figure 7 (left) shows the result of a Hin reaction time course in which pRJ858 (DR) was nicked with DNase I to remove supercoils prior to electrophoresis. A series of knots containing from 3 to $>15$ nodes is generated with increasing time. The primary knotted products are multiples of three, giving rise to 3-, 6-, 9-, 12-, and 15-noded molecules. In addition, an increasing number of molecules with 5, 8, 11, and 14 nodes are also formed. The pattern of knots in pRJ858 (DR) corresponds precisely to those generated in pRJ862 (IR), which contains a mutation within the core nucleotides of one recombination site (Fig. 7 (right); Heichman et al., this issue). This demonstrates that substrates with directly repeated recombination sites and substrates containing inverted recombination sites with core mutations are equally as reactive for knotting. These results lead to a model in which plasmids containing directly repeated sites are ef-
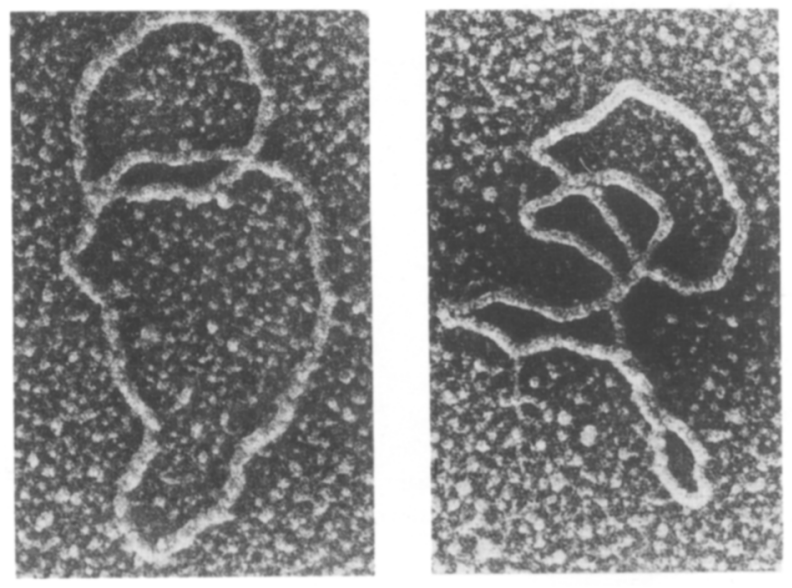

Figure 6. Electron microscopy of RecA-coated catenated deletion products. RecA-coated pRJ864 (DR) molecules are shown, demonstrating that the products of deletion are singly linked catenanes. The sizes of the interlinked DNA molecules are consistent with a deletion between the two recombination sites of pRJ864, generating a 1690-bp molecule containing the enhancer and a 3780-bp vector sequence.

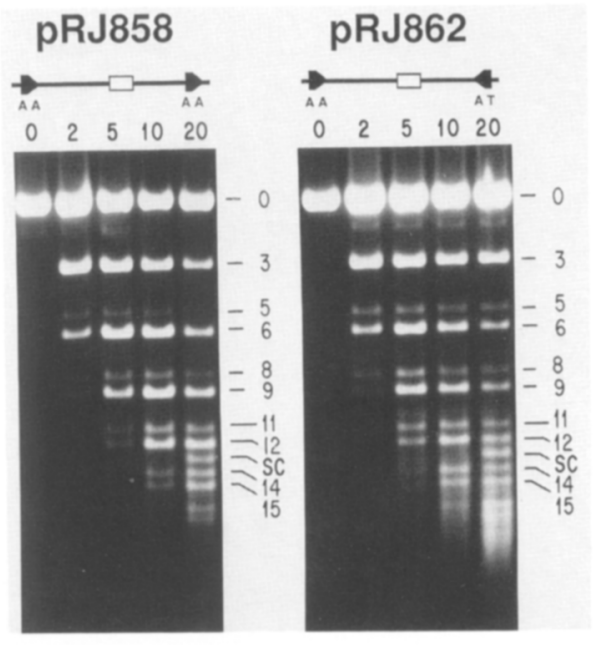

Figure 7. Hin-mediated DNA knotting of pRJ858 (DR) and pRJ862 (IR). pRJ858 (DR) and pRJ862 (IR) were incubated with Hin, Fis, and $\mathrm{HU}$ for $0,2,5,10$, and $20 \mathrm{~min}$, as indicated, nicked with DNase I, and electrophoresed in an agarose gel. Knots of increasing complexity migrate faster in the gel. The number of nodes in each species is indicated at the right of each panel. The node assignments for pRJ858 (DR) are based on those determined for pRJ862 (IR) (see Heichman et al., this issue) and by electrophoresing a knot ladder generated by phage T4 topoisomerase in parallel.

ficiently forming a recombination complex that is identical to that formed normally between inverted sites (see Figs. $1 \mathrm{D}$ and $8 \mathrm{~B}$ ). In this assembly, a single $180^{\circ}$ rotation will position the core nucleotides into a mismatched configuration. Therefore, an additional rotation leading to the formation of a three-noded knot would be required to achieve ligation once strand exchange has been initiated. Two additional rotations will generate a five-noded product, and multiple independent reactions will lead to more complex knots (Heichman et al., this issue).

\section{Recombination between substrates containing symmetric core nucleotides}

The model described above suggests that plasmids containing their recombination sites in directly repeated orientation assemble efficiently into an invertasome complex in which the recombination sites are in a reverse or antiparallel orientation. The inability to invert the intervening DNA between directly repeated sites is primarily or exclusively a function of the nonidentity of the core nucleotides, which prevent ligation after a $180^{\circ}$ rotation. This model was tested with recombination sites containing a symmetric 2-bp core sequence. In this case, ligation after a single $180^{\circ}$ rotation of strands could be achieved regardless of the starting arrangement of recombination sites at synapsis (see Fig. 8C). Thus, a plasmid with two recombination sites containing symmetric core sequences in directly repeated configuration should support a high rate of inversion, as well as a low rate of deletion.

Plasmids were constructed with both recombination 


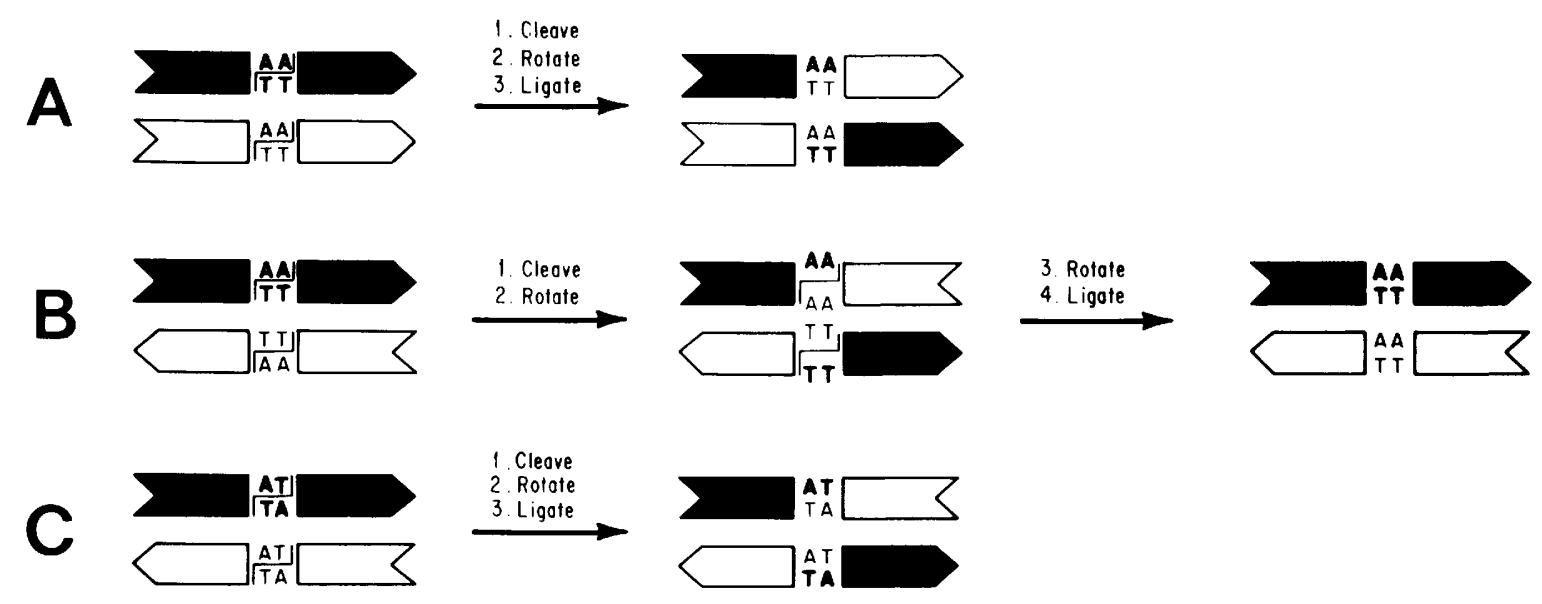

Figure 8. Synapsis and strand exchange of recombination sites in inverted and directly repeated orientation. $(A)$ Two wild-type recombination sites (solid and open) in inverted orientation on a DNA molecule are aligned together at synapsis as in pathway $A$ in Fig. 1. A staggered cleavage within the core nucleotides, followed by exchange of the half-sites and ligation, as shown, results in inversion of the intervening DNA segment. $(B)$ Two wild-type recombination sites in a directly repeated configuration on a DNA molecule are aligned together in reverse (antiparallel) orientation at synapsis as in pathway $D$ in Fig. 1 . DNA cleavage and exchange as in $A$ generates non-base-paired core sequences that cannot ligate efficiently. An additional exchange restores the parental configuration of the DNA but generates a three-noded knot. $(C)$ Two mutant (hix $L-A T)$ recombination sites in directly repeated configuration on a DNA molecule are aligned together in reverse orientation at synapsis as in $B$. In this case, DNA cleavage and a single exchange results in core sequences that can base-pair and thus ligate efficiently, generating inversion of the intervening DNA segment.

sites containing AT for their core nucleotides (hix $L-A T)$. The recombination sites were positioned in either inverted or directly repeated orientation, and the enhancer was placed either close to one recombination site [pMS631 (IR) and pRJ857 (DR)] or well spaced from both recombination sites [pRJ863 (IR) and pRJ864 (DR)] (Fig. 2). Each of these plasmids was assayed in vitro and in vivo for their ability to support inversion or deletion. High rates of inversion, which were similar to those in plasmids containing hix $L-W T$ sites in inverted orientation, were measured for the plasmids containing hixL$A T$ sites in either orientation (Fig. 9; Table 1; data not shown). Each of the hixL-AT plasmids also supported a low rate of deletion that was similar to plasmids containing hix $L-W T$ sites in direct repeat (Fig. 10; Table 1; data not shown). No deletion or inversion products were observed in plasmids containing hixL-WT sites in inverted or direct repeat orientation, respectively. These results clearly demonstrate the importance of the core nucleotides in determining the product of recombination but indicate that the relative orientation of the recombination sites is not important for reactivity.

The apparent preference for assembly of recombination sites into the invertasome structure, regardless of the sequence of the recombination sites, was investigated further by assaying the product of recombination with a plasmid containing hix sites that are symmetric over their entire sequence. pMS656 has recombination sites with a palindromic sequence that is a consensus of the DNA invertase family (hixC; see Fig. 2B). Because the recombination sites are completely symmetric, their relative orientation cannot be distinguished. Although pMS656 does not recombine as efficiently as the plasmids with hix $L-A T$ sites, a $>10$-fold bias of inversion over deletion is measured in vitro (data not shown) and in vivo (Table 2). These results argue further that the nucleotide sequence of the recombination site outside of the core sequence is not responsible for mediating directionality.

\section{Discussion}

We have characterized the Hin-promoted deletion reaction that excises DNA between two hix sites. The deletion reaction is much less efficient than inversion. In the presence of moderately overproduced Hin protein, the rate of deletion in vivo is $<10 \%$ of the rate of inversion in plasmids with appropriately oriented recombination sites. The difference in the relative rates of the two reactions is even more pronounced in vitro, where deletions are produced at $0.3 \%$ of the rate measured for inversion. In vitro experiments have shown that the deletion reaction requires DNA supercoiling, the enhancer DNA segment, and Fis, suggesting that the nucleoprotein complex that is assembled to promote deletion is similar to the invertasome structure that is the precursor of inversion. The most significant difference is the requirement for additional loops of DNA to orient directly repeated recombination sites into a configuration that will yield deletion. On the basis of topological constraints imposed by supercoiling and the finding that the deletion products are singly catenated, the loops are likely to be positioned as depicted in Figure 1B.

\section{Determinants of recombination site alignment}

Does the low rate of deletion reflect the inefficiency of two sites in directly repeated configuration to be effi- 


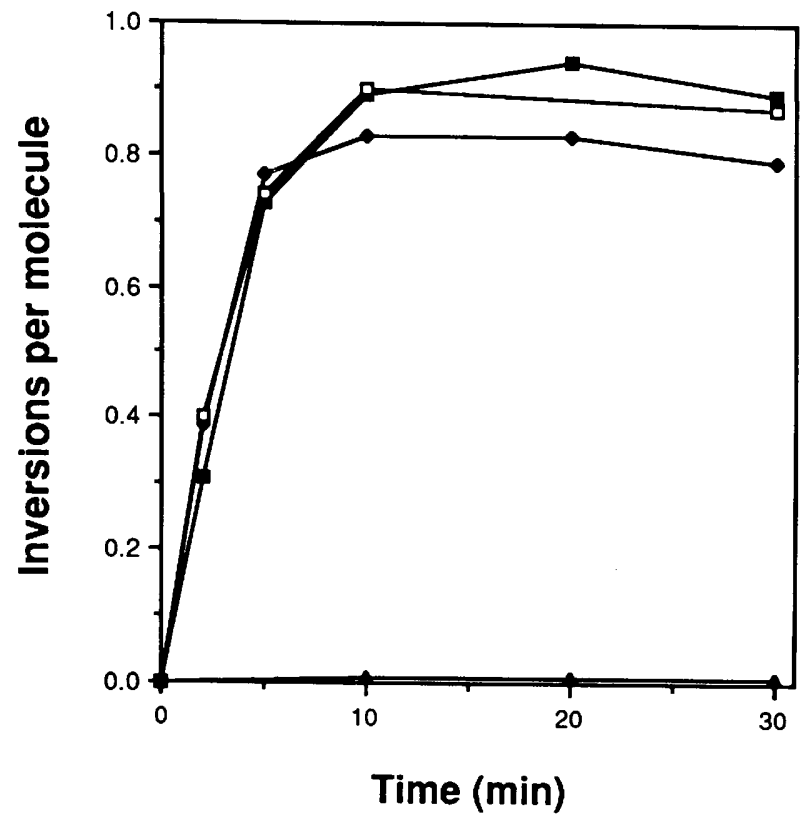

Figure 9. Rates of in vitro inversion of wild-type and mutant substrates. pMS634 (IR) (•), pRJ863 (IR) (D), pRJ864 (DR) (ロ), and pRJ858 (DR) ( $\mathbf{\Delta})$ were incubated with Hin, Fis, and HU for various times, digested with restriction enzymes, and electrophoresed in an agarose gel to determine the fraction of molecules containing inversions. The results are plotted as inversions per substrate molecule as a function of time. pMS634 (IR) and pRJ858 (DR) contain two hixL-WT sites, and pRJ863 and pRJ864 contain two hix $L-A T$ sites.

ciently recognized or assembled into a reactive complex? This cannot be the case for several reasons. A plasmid substrate containing the wild-type recombination sites in directly repeated configuration supports an efficient knotting reaction with rates similar to that observed with a substrate containing inverted sites with nonhomologous core residues. Most importantly, plasmids containing directly repeated sites with symmetric core nucleotides undergo an efficient inversion reaction, as well as an inefficient deletion reaction. The combined results indicate that recombination sites in directly repeated orientation are efficiently assembled into an invertasome complex that is capable of initiating strand exchange.

The overwhelming propensity for assembly of the Hin recombination sites into a configuration that leads to inversion may be due exclusively to constraints placed by the conformation of the supercoiled DNA substrate. Two mechanisms may be contributing to the biased assembly of recombination sites; these mechanisms are not necessarily mutually exclusive. The first is that a particular juxtaposition or interwrapping of proteinbound DNA sites is favored on supercoiled DNA /Craigie and Mizuuchi 1986; Boocock et al. 1987; Gellert and Nash 1987; Stark et al. 1989). This interwrapping may be required to successfully assemble a complex competent to promote recombination and will not favor formation of a deletion complex. The second is that plectonemically wound DNA strands that are continually sliding past each other would preferentially generate collisions between sites in a configuration that would lead to inversion upon strand exchange (Benjamin and Cozzarelli 1986). The association of sites may occur in a branched DNA molecule to directly give the invertasome structure that contains the enhancer, or more likely, the two recombination sites may first assemble into a complex prior to association with the enhancer. Both types of complexes have been observed by electron microscopy, and their formation has been shown to be strongly stimulated or dependent on DNA supercoiling (Heichman and Johnson 1990). For recombination sites in inverted orientation, the favored (parallel) alignment of sites will generate inversion products upon a single $180^{\circ}$ rotation (Figs. 1A and 8A). For recombination sites in direct repeat orientation, the sites will join in an antiparallel alignment as in pathway D of Figure 1. A single $180^{\circ}$ rotation of strands results in core sequences that are unable to base-pair (Fig. 8B). A second rotation is required to allow for base-pairing and ligation. This leaves the primary structure of the DNA unchanged; however, because of the geometry of the DNA strands in the invertasome, a knot is generated. Alignment of the recombination sites into a configuration that will lead to singly catenated deletion products necessitates that one of the sites be turned $180^{\circ}$, generating the structures depicted in Figure 1, pathway B. The assembly of these structures would not be kinetically favored on supercoiled DNA, and the structures themselves would be under severe torsional stress that would thermodynamically discourage the same interwrapping of sites found in the inversion complexes.

It was surprising to find that a large length of DNA between the enhancer and both recombination sites was required for the in vitro deletion reaction. We would predict that the additional looping required for the deletion intermediate would occur on the DNA segment of

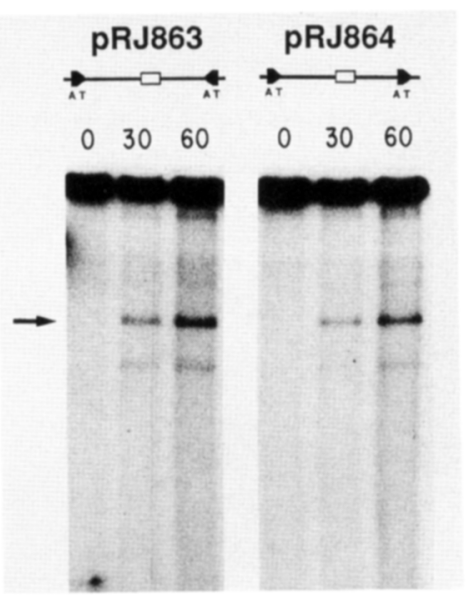

Figure 10. In vitro deletion of mutant substrates. pRJ863 (IR) and pRJ864 (DR) were incubated with Hin, Fis, and HU for 0, 30, and $60 \mathrm{~min}$, digested with $N d e \mathrm{I}$, which cuts once in the vector, end-labeled with ${ }^{32} \mathrm{P}$, electrophoresed in an agarose gel, and autoradiographed. The arrow indicates the location of the labeled deletion product. 
greater length. However, even with one large segment of DNA between the enhancer and a recombination site, very low deletion rates were observed if the other segment was at the wild-type spacing. It is possible that stress placed on the deletion intermediate structure by the additional looping of DNA may distort the structure sufficiently to inhibit association of the enhancer with the second recombination site separated by a short DNA segment. The effect of DNA length on deletion rates can be partially overcome by additional HU. In vivo, the requirement for large segments of DNA between both recombination sites and the enhancer is not observed. The interaction of DNA in vivo with chromosomal binding proteins like HU, perhaps together with the reduced superhelical tension of DNA in the cell (Sinden et al. 1983; Bliska and Cozzarelli 1987), may relieve some of the topological stress in the deletion complex. These factors may also contribute to the reduced preference of inversions over deletions measured in vivo as compared with in vitro.

We conclude that the product of Hin-mediated recombination is governed by two factors. The first is the choice of assembly of recombination sites into a nucleoprotein complex to yield inversion or deletion of the intervening DNA. Probably because of the nature of supercoiled DNA, the sites preferentially assemble into a configuration that yields inversion. Once a recombination complex is assembled, the sequence of the core residues determines whether an attempt at inversion or deletion will be successful. Ability of the strands to ligate following an initial $180^{\circ}$ rotation results in the formation of recombinants. If the DNA strands cannot be ligated after a single $180^{\circ}$ rotation of strands, further rotations in the same direction can occur to achieve ligation; however, this results in the formation of knots in the DNA. A similar mechanism for a reaction between directly repeated sites has been proposed independently for the Gin inversion system (Kanaar et al. 1990).

Recent analysis of another related site-specific recombination system, resolution by $\mathrm{Tn} 3$ resolvase, has provided valuable insight into recombination site alignment. The resolvase system displays a strong preference for a particular configuration of sites that gives rise to resolution over inversion (Reed and Grindley 1981; Krasnow and Cozzarelli 1983), just as the invertases show such a strong preference for inversion over deletion. Unlike Hin, however, resolvase does not require any accessory proteins such as Fis, nor does it require a recombinational enhancer. The res recombination sites are more complicated than hix sites, containing three resolvase protein-binding subsites (Grindley et al. 1982). These additional binding sites have been shown to dictate the strict specificity of resolvase-mediated recombination (Dröge and Cozzarelli 1989; Stark et al. 1989). Thus, like the Hin system, synapsis of the res recombination sites is not determined exclusively by a particular parallel or antiparallel orientation (Bednarz et al. 1990). Rather, alignment is dependent on the preferential formation of a particular synaptic intermediate, which leads to the bias of one recombinant product over the other.

\section{Implications for in vivo recombination by Hin}

The structure of the hix recombination site is relatively simple, particularly in comparison with other site-specific recombination sites utilized in systems such as $\lambda$ and resolvase. It has been observed that Hin can bind to a variety of pseudo-hix sites (Glasgow et al. 1989) and, in some cases, can recruit these sites into a recombination complex that can initiate cleavage (Johnson and Bruist 1989; Heichman and Johnson 1990). The only criterion left for the system to determine whether the chosen sites will recombine to change the primary structure of the DNA is the identity of the 2-bp core nucleotides. Given random sequence, recombination will be productive 1 of every 16 times. The remainder of the events will undergo an additional rotation to restore the original sequence. This may lead to an unacceptably high rate of Hin-promoted chromosomal rearrangements. However, several additional levels of control operate to limit unwanted rearrangements. Hin protein is synthesized at an extremely low level, and it has been shown that the amount of Hin limits the rate of the inversion reaction controlling $\mathrm{H} 2$ flagellin expression (Bruist and Simon 1984). In addition, the Hin protein cannot efficiently catalyze inversion or deletion without the presence of a recombinational enhancer sequence located somewhat nearby. The maximum distance allowed for function of the recombinational enhancer is not known, but the observation that inversion rates are reduced by threefold in vitro when the enhancer is $4 \mathrm{~kb}$ from the closest hix site suggests that it may not function over extremely great distances (Johnson et al. 1986). The constraints on the structure of the recombinational enhancer (two Fis-binding sites separated by 48 or $58 \mathrm{bp}$ ) would limit the number of these sites present in the chromosome (Johnson et al. 1987). Finally, the potential biological consequences of a deletion (the less favored reaction) would be far greater than an inversion, which will only cause mutations at the site of recombination on the chromosome.

\section{Materials and methods}

Enzymes and reagents

Purified Hin, Fis, and HU preparations were obtained as described in Heichman et al. (this issue). Restriction enzymes and isopropylthio- $\beta$-D-galactoside (IPTG) were purchased from Boehringer Mannheim or New England Biolabs; AMV reverse transcriptase was from Promega; and calf thymus topoisomerase I was from Bethesda Research Laboratories, Life Technologies, Inc. Antibiotics were obtained from Sigma.

\section{In vitro Hin recombination reactions}

The reaction conditions used to generate inversions, deletions, and knots were identical except where noted and are described (Heichman et al., this issue). Five percent dimethylsulfoxide was included in reactions with pMS656. Inversions were detected by digestion of the reacted plasmids with the appropriate restriction enzymes to determine the orientation of the invertible segment followed by agarose gel electrophoresis. Deletion products were typically detected as follows: Reacted plasmids were digested with a restriction enzyme that cleaves the DNA 
once within the vector or deletion segment and then end-filled with $10 \mu \mathrm{Ci}\left[\alpha^{-32} \mathrm{P}\right] \mathrm{dATP}$ (Amersham, $>3000 \mathrm{Ci} / \mathrm{mmole}$ ) with AMV reverse transcriptase. The labeled DNA molecules were detected by autoradiography after agarose gel electrophoresis. The reactions were quantitated by Cerenkov counting of gel slices or densitometry of autoradiographs with an LKB Ultroscan model 2202 laser densitometer, and the peak values were integrated with the LKB 2190 version 5.2 GelScan software package. Knots were analyzed as described (Heichman et al., this issue).

\section{In vivo Hin recombination reactions}

In vivo recombination reactions were performed in RJ2626 [pRJ918, pRJ823, $\Delta$ (lac-pro) recA56 rpsL ara srl]. This strain contains the hin gene tightly regulated by the lac promoter on pRJ918 such that under standard conditions, no inversion or deletion is detectable until IPTG is added. Substrate plasmids were transformed into RJ2626 and plated on LB agar containing $100 \mu \mathrm{g} / \mathrm{ml}$ of ampicillin, $15 \mu \mathrm{g} / \mathrm{ml}$ of tetracycline, $50 \mu \mathrm{g} / \mathrm{ml}$ of spectinomycin, and $0.2 \%$ glucose. Single colonies appearing after overnight growth were inoculated into LB plus antibiotics and grown at $37^{\circ} \mathrm{C}$ until $\mathrm{OD}_{600}=0.3$ at which time IPTG was added to $1 \mathrm{~mm}$. Aliquots were taken at appropriate times, and plasmid DNA was isolated by the alkaline-SDS lysis method (Maniatis et al. 1982). Cultures were maintained in exponential phase by continual dilution with prewarmed media. Inversions or deletions were detected as described above.

\section{Plasmid structures and constructions}

The overall structure of each of the substrate plasmids used in this study is similar. The plasmids, which are all derivatives of pBR322, have one recombination site located at the EcoRI site and one recombination site located at the SalI site. The relative orientations of the two recombination sites and the locations of the enhancer segment are depicted in Figure 2. The details of the structures and constructions of the plasmids are described be low. pMS551, pMS631, and pMS634 have been described previously (Johnson and Simon 1985). pMS638 and pRJ857 are identical to pMS551 and pMS631, respectively, except that the hixL sequence at the Sall site is oriented in directly repeated orientation with respect to the hixL at the EcoRI site. pRJ858 was constructed by a ligation of the EcoRI-HindIII DNA fragment from pMS634, which contains hixL plus the enhancer with the EcoRI-HindIII fragment from pMS638, which contains the pBR322 ori and hixL. pRJ907 was made from pRJ858 by deleting the 87-bp segment of DNA between the ClaI site in the enhancer and the unique HindIII site. This removes the distal domain and most of the intervening DNA between the two domains of the enhancer. The construction of pRJ862 is described in Heichman et al. (this issue). pRJ863 and pRJ864 are derived from pMS631 and pRJ857, respectively, by inserting into the SmaI sites of these plasmids a 789-bp HindII fragment containing the lacPL162 mutation (Reznikoff et al. 1982). The same 789-bp fragment was ligated into an end-filled HindIIl site in pMS638 to give pRJ974.

pMS656 was constructed as follows: The oligonucleotide d/TTATCCTAAACCATGGTTTAGGATAA|, encoding a consensus and completely symmetric recombination site (hixC), was ligated into the SmaI site of M13mp8 to give mMS500. The EcoRI-BamHI fragment containing hixC was ligated into similarly cut pMS577 (Johnson and Simon 1985), which contains the enhancer, to give pMS655. The EcoRI-SalI fragment containing hix C from mMS500 was also ligated into the SalI site of pBR322 with T4 DNA polymerase to fill in the noncomplemen- tary ends to give pMS653. pMS656 was constructed from these two plasmids by substituting the EcoRI-HindIII fragment from pMS655, which contains hixC plus the enhancer for the 29-bp EcoRI-HindIII region in pMS653.

pRJ823 (lacI ${ }^{q}, \mathrm{p} 15 \mathrm{~A}$ ori, tet $)$ was generated by transferring the EcoRI fragment containing the $1 a c I^{q}$ gene from pMS421 (M. Susskind, University of Southern California) into the EcoRI site of pACYC184 (Chang and Cohen 1978). pRJ918 contains the lacPO region fused to the hin gene $40 \mathrm{bp}$ before the initiating AUG in a low-copy-number replicon. It was constructed by ligating a $P$ vuII-HindIII fragment containing lacPO-hin from pMS568 (Johnson and Simon 1985) into the SmaI-HindIII linker region of pMS421 (lacI ${ }^{q}, \mathrm{pSCl} 101$ ori, spc/str).

\section{Electron microscopy of deletion products}

RecA coating and electron microscopy of DNA molecules was performed as described in Heichman et al. (this issue). pRJ864 or pMS631 was reacted for $30 \mathrm{~min}$ and electrophoresed through agarose gels after nicking with DNase I. The DNA that migrated faster than the parental nicked species was excised, purified with the Geneclean protocol (Bio 101), and coated with RecA protein.

\section{Acknowledgments}

We thank C. Ball and R. Osuna for their helpful comments on the manuscript. This work was supported by grant GM38509 from the National Institutes of Health and the Searle Scholars Program/The Chicago Community Trust. K.A.H. was supported in part by U.S. Public Health Service National Institutional Research Service Award CA-09056 and a Dr. Ursula Mandel Scholarship.

The publication costs of this article were defrayed in part by payment of page charges. This article must therefore be hereby marked "advertisement" in accordance with 18 USC section 1734 solely to indicate this fact.

\section{References}

Bednarz, A.L., M.R. Boocock, and D.J. Sherratt. 1990. Determinants of correct res site alignment in site-specific recombination by Tn3 resolvase. Genes \& Dev. 4: 2366-2375.

Benjamin, H.W. and N.R. Cozzarelli. 1986. DNA-directed synapsis in recombination: Slithering and random collision of sites. Proc. Robert A. Welsh Found. Conf. Chem. Res. 29: 107-126.

Bliska, J.B. and N.R. Cozzarelli. 1987. Use of site-specific recombination as a probe of DNA structure and metabolism in vivo. J. Mol. Biol. 194: 205-218.

Boocock, M.R., J.L. Brown, and D.J. Sherratt. 1987. Topological specificity in Tn3 resolvase catalysis. In DNA replication and recombination (eds. T.J. Kelly and R. McMacken), pp. 703-718. Alan R. Liss, New York.

Bruist, M.F. and M.I. Simon. 1984. Phase variation and the Hin protein: In vivo activity measurements, protein overproduction and purification. J. Bacteriol. 159: 71-79.

Bruist, M.F., A.C. Glasgow, R.C. Johnson, and M.I. Simon. 1987. Fis binding to the recombinational enhancer of the Hin DNA inversion system. Genes \& Dev. 1: 762-772.

Chang, A.C.Y. and S.N. Cohen. 1978. Construction and characterization of amplifiable multicopy DNA cloning vehicles derived from the P15A cryptic miniplasmid. J. Bacteriol. 134: 1141-1156.

Craigie, R. and K. Mizuuchi. 1986. Role of DNA topology in Mu 
transposition: Mechanism of sensing the relative orientation of two DNA segments. Cell 45: 795-800.

Dröge, P. and N.R. Cozzarelli. 1989. Recombination of knotted substrates by Tn3 resolvase. Proc. Natl. Acad. Sci. 86: 60626066.

Gellert, M. and H. Nash. 1987. Communication between segments of DNA during site-specific recombination. Nature 325: 401-404.

Glasgow, A.C., M.F. Bruist, and M.I. Simon. 1989. DNA-binding of the Hin recombinase. J. Biol. Chem. 264: 1007210082.

Grindley, N.D.F., M.R. Lauth, R.G. Wells, R.J. Wityk, J.J. Salvo, and R.R. Reed. 1982. Transposon-mediated site-specific recombination: Identification of three binding sites for resolvase at the res sites of $\gamma \delta$ and Tn3. Cell 30: 19-27.

Heichman, K.A. and R.C. Johnson. 1990. The Hin invertasome: Protein-mediated joining of distant recombination sites at the enhancer. Science 249: 511-517.

Iida, S., J. Meyer, K. Kennedy, and W. Arber. 1982. A site-specific conservative recombination system carried by bacteriophage P1. EMBO I. 1: 1445-1453.

Johnson, R.C. and M.F. Bruist. 1989. Intermediates in Hin-mediated DNA inversion: A role for Fis and the recombinational enhancer in the strand exchange reaction. EMBO J. 8: $1581-1590$.

Johnson, R.C. and M.I. Simon. 1985. Hin-mediated site-specific recombination requires two 26-bp recombination sites and a 60-bp recombinational enhancer. Cell 41: 781-789.

Johnson, R.C., M.F. Bruist, M.B. Glaccum, and M.I. Simon. 1984. In vitro analysis of Hin-mediated site-specific recombination. Cold Spring Harbor Symp. Quant. Biol. 49: 751760.

Johnson, R.C., M.F. Bruist, and M.I. Simon. 1986. Host protein requirements for in vitro site-specific DNA inversion. Cell 46: $531-539$.

Johnson, R.C., A.C. Glasgow, and M.I. Simon. 1987. Spatial relationship of the Fis binding sites for Hin recombinational enhancer activity. Nature 329: 462-465.

Kamp, P., R. Kahmann, D. Zipser, T.R. Broker, and L.T. Chow. 1978. Inversion of the G DNA segment of phage $\mathrm{Mu}$ controls phage infectivity. Nature 271: 577-580.

Kanaar, R., A. Klippel, E. Shekhtman, J.M. Dungan, R. Kahmann, N.R. Cozzarelli. 1990. Processive recombination by the phage Mu Gin system: Implications for the mechanisms of DNA strand exchange, DNA site alignment, and enhancer action. Cell 62: 353-366.

Kennedy, K.E., S. Iida, J. Meyer, M. Stalhammar-Carlemalm, R. Hiestand-Nauer, and W. Arber. 1983. Genome fusion mediated by the site-specific DNA inversion system of bacteriophage P1. Mol. Gen. Genet. 189: 413-421.

Koch, C. and R. Kahmann. 1986. Purification and properties of the Escherichia coli host factor required for inversion of the G segment in bacteriophage Mu. I. Biol. Chem. 261: 1567315678.

Krasnow, M.A. and N.R. Cozzarelli. 1983. Site-specific relaxation and recombination by the Tn3 resolvase: Recognition of the DNA path between orientated sites. Cell 32: 13131324.

Krasnow, M.A., A. Stasiak, S.J. Spengler, F. Dean, T. Koller, and N.R. Cozzarelli. 1983. Determination of the absolute handedness of knots and catenanes of DNA. Nature 304: 559560.

Maniatis, T., E.F. Fritsch, and J. Sambrook. 1982. Molecular cloning: A laboratory manual, pp. 368-369. Cold Spring Harbor Laboratory, Cold Spring Harbor, New York.

Plasterk, R.H.A., T. Ilmer, and P. van de Putte. 1983. Site-spe- cific recombination by Gin of bacteriophage $\mathrm{Mu}$ : Inversions and deletions. Virology 127: 24-36.

Reed, R.R. and N.D.F. Grindley. 1981. Transposon-mediated site-specific recombination in vitro: DNA cleavage and protein-DNA linkage at the recombination site. Cell 25: 721728.

Reznikoff, W.S., L.E. Maquat, L.E. Munson, R.C. Johnson, and W. Mandecki. 1982. The lac promoter: Analysis of structural signals for transcription initiation and identification of a new sequence specific event. In Promoters: Structure and function (ed. R.L. Rodriguez and M.J. Chamberlin), pp. 8095. Praeger Publishers, New York.

Scott, T.N. and M.I. Simon. 1982. Genetic analysis of the mechanism of the Salmonella phase variation site-specific recombination system. Mol. Gen. Genet. 188: 313-321.

Silverman, M. and M. Simon. 1980. Phase variation: Genetic analysis of switching mutants. Cell 19: 845-854.

Sinden, R.R., S.S. Broyles, and D.E. Pettijohn. 1983. Perfect palindromic lac operator DNA sequence exists as a stable cruciform structure in supercoiled DNA in vitro but not in vivo. Proc. Natl. Acad. Sci. 80: 1797-1801.

Stark, W.M., D.J. Sherratt, and M.R. Boocock. 1989. Site-specific recombination by $\mathrm{Tn} 3$ resolvase: Topological changes in the forward and reverse reactions. Cell 58: 779-790.

Zieg, J. and M. Simon. 1980. Analysis of the nucleotide sequence of an invertible controlling element. Proc. Natl. Acad. Sci. 77: 4196-4200. 


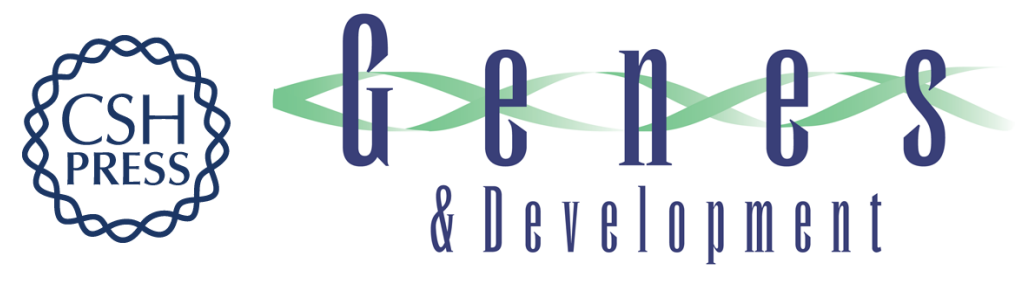

\section{Alignment of recombination sites in Hin-mediated site-specific DNA recombination.}

I P Moskowitz, K A Heichman and R C Johnson

Genes Dev. 1991, 5:

Access the most recent version at doi:10.1101/gad.5.9.1635

References This article cites 31 articles, 11 of which can be accessed free at: http://genesdev.cshlp.org/content/5/9/1635.full.html\#ref-list-1

License

Email Alerting

Receive free email alerts when new articles cite this article - sign up in the box at the top Service right corner of the article or click here.

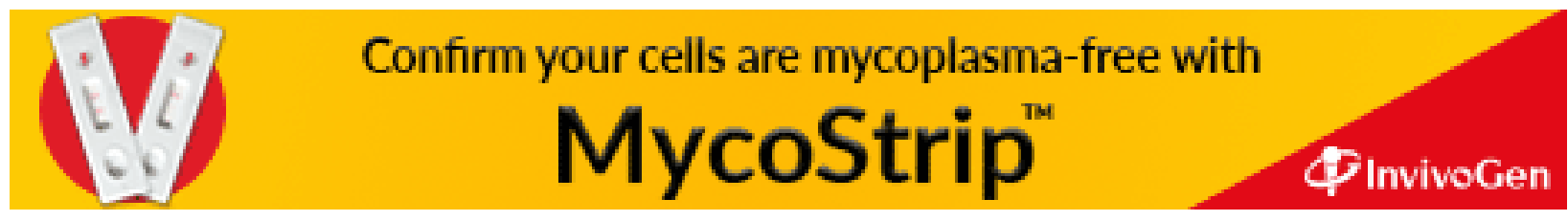

